BNL-112734-2016-JA

\title{
Direct and real time probe of photoinduced structure transition in colossal magnetoresistive material
}

\author{
Junjie Li, Xuan Wang, Haidong Zhou, \\ Jun Zhou, J. G. Cheng, Jianming Cao
}

Submitted to Applied Physics Letters

July 2016

\section{Condensed Matter Physics and Material Science Department \\ Brookhaven National Laboratory}

\author{
U.S. Department of Energy \\ USDOE Office of Science (SC), \\ Basic Energy Sciences (BES) (SC-22)
}

Notice: This manuscript has been authored by employees of Brookhaven Science Associates, LLC under Contract No. DE-SC0012704 with the U.S. Department of Energy. The publisher by accepting the manuscript for publication acknowledges that the United States Government retains a non-exclusive, paid-up, irrevocable, world-wide license to publish or reproduce the published form of this manuscript, or allow others to do so, for United States Government purposes. 


\section{DISCLAIMER}

This report was prepared as an account of work sponsored by an agency of the United States Government. Neither the United States Government nor any agency thereof, nor any of their employees, nor any of their contractors, subcontractors, or their employees, makes any warranty, express or implied, or assumes any legal liability or responsibility for the accuracy, completeness, or any third party's use or the results of such use of any information, apparatus, product, or process disclosed, or represents that its use would not infringe privately owned rights. Reference herein to any specific commercial product, process, or service by trade name, trademark, manufacturer, or otherwise, does not necessarily constitute or imply its endorsement, recommendation, or favoring by the United States Government or any agency thereof or its contractors or subcontractors. The views and opinions of authors expressed herein do not necessarily state or reflect those of the United States Government or any agency thereof. 


\title{
Direct and real time probe of photoinduced structure transition in colossal magnetoresistive material
}

\author{
Junjie Li, ${ }^{1,2,3}$ Xuan Wang, ${ }^{4}$ Haidong Zhou, ${ }^{2}$ Jun Zhou, ${ }^{1,2}$ J. G. Cheng, ${ }^{5}$ \\ Jianming Cao ${ }^{1,2, \text { a) }}$
}

${ }^{1}$ Physics Department, Florida State University, Tallahassee, Florida 32310, USA

${ }^{2}$ National High Magnetic Field Laboratory, Tallahassee, Florida 32310, USA

${ }^{3}$ Condensed Matter Physics and Materials Science Department, Brookhaven National Laboratory, Upton, NY 11973, USA

${ }^{4}$ Institute of physics, Chinese Academy of Sciences, P.O.Box 603, Beijing 100190, China

${ }^{5}$ Texas Materials Institute, University of Texas at Austin, Austin, Texas 78712, USA

\begin{abstract}
We report a direct and real time measurement of photoinduced structure phase transition in single crystal $\mathrm{La}_{0.84} \mathrm{Sr}_{0.16} \mathrm{MnO}_{3}$ using femtosecond electron diffraction. The melting of orthorhombic lattice ordering under femtosecond optical excitation is found involving two distinct processes with different time scales, an initial fast melting of orthorhombic phase in about 4 ps and a subsequent slower transformation in 90 ps and longer timescales. The fast process is designated as the initial melting of orthorhombic phase induced by the Mn-O bond change that is most likely driven by the quenching of the dynamic Jahn-Teller distortion following the photo-excitation. The slow process is attributed to the growing of newly formed structure domain from the photo-excited sites to the neighboring non-excited orthorhombic sites.
\end{abstract}

\footnotetext{
${ }^{a}$ Author to whom correspondence should be addressed

Electronic address: jcao@magnet.fsu.edu
} 
Colossal Magnetoresistance (CMR) refers to a huge electrical resistance change with the application of a magnetic field in manganese-based perovskite oxides. The CMR effect is most pronounced around the Curie temperature where the ferromagnetic, metal-insulator and lattice structure phase transitions take place. ${ }^{1-3}$ Extensive theoretical and experimental studies show that charge, spin and lattice in CMR materials are strongly coupled to one another, and the interplay among these subsystems is not only responsible for the CMR effect but also creating a rich phase diagram with multiple competing phases. An in-depth understanding of the interactions among different subsystems and the dynamics of phase transitions between different competing phases is crucial to understand the rich physics, such as CMR effect, and to manipulate the magnetic properties in application. ${ }^{2-7}$ For many CMR materials, the transitions among competing phases can be triggered by ultrafast optical excitation, ${ }^{8-11}$ providing a mean to interrogate their dynamical processes in time domain with ultrafast techniques. These time-domain methods hold the promise to separate the complicated interactions among subsystems in time domain, so that the role of each subsystem could be singled out and interrogated individually. Nonetheless, direct and real time observation of photoinduced structure phase transition in CMR materials has been sparse so far.

In this study, we report a direct and real-time measurement of photoinduced structure phase transition in a typical CMR material $\mathrm{La}_{1-\mathrm{x}} \mathrm{Sr}_{\mathrm{x}} \mathrm{MnO}_{3}$ (LSMO) with femtosecond electron diffraction (FED). Within a limited doping range, LSMO crystal undergoes a reversible structural phase transition from rhombohedral to orthorhombic ordering with decreasing temperature. ${ }^{12,13}$ Particularly, at a doping level of $\mathrm{x}=0.160$, its structure is orthorhombic below $325 \mathrm{~K}$, rhombohedral above $340 \mathrm{~K}$ and bi-stable in between. Previous studies show that this structure phase transition could also be triggered by magnetic field ${ }^{3}$ and is accompanied with an abnormal electrical resistance change, ${ }^{14}$ indicating the strong coupling among different sub-systems. In terms of structure, a major difference between these two phases is that orthorhombic symmetry has a superlattice structure with a lattice constant almost twice that of the parent perovskite unit cell along the [001] direction, but none in the rhombohedral phase. ${ }^{15}$ Therefore, superlattice diffraction spots will exist in the electron diffraction pattern (DP) of its orthorhombic structure, and their disappearance represents the orthorhombic-to- 
rhombohedral structure phase transition in static measurements. ${ }^{13}$ Using femtosecond electron diffraction with a sub-ps temporal resolution, we are able to explore the possibility of using photon to induce this orthorhombic-to-rhombohedral phase transition and recording the associated structure change in real time, ${ }^{16-18}$ thus gaining insights into the transition dynamics.

Single crystal LSMO samples were grown in a floating zone furnace. ${ }^{19,20}$ The crystal was first checked with X-ray Laue diffraction to determine the crystal orientation and then cut into thin discs along the [100] direction. After being mechanically polished to a thickness of $\sim 30 \mu \mathrm{m}$, each disc was further thinned to be electron transparent $(<100 \mathrm{~nm})$ by means of $\mathrm{Ar}^{+}$ion milling. Before being put into the ultra-high vacuum chamber to study the photoinduced phase transition, the sample was carefully examined by TEM to ensure high quality and uniform microscopic structure across a large area of a few hundred microns. The overall FED setup is similar to our earlier experiments ${ }^{21}$ and the relevant geometry of the pump-probe pulses and the LSMO sample is drawn in Fig. 1 (a). Before time-resolved FED measurements, the orthorhombic-to-rhombohedral phase transition has been verified for the LSMO samples used in this study by running femtosecond electron diffraction in the static mode, in which the sample temperature is set by a heating-cooling stage and DPs are taken with the pump optical beam blocked. In time-resolved measurements, pump optical pulses with a duration of $50 \mathrm{fs}$ and a center wavelength of $800 \mathrm{~nm}$ were focused down to $\sim 2 \mathrm{~mm}$ on the electron-transparent area of the sample, producing sufficient fluence to trigger the structure phase transition. Wellsynchronized electron pulses of 300 fs temporal width at $88 \mathrm{keV}$ beam energy were collimated down to $300 \mu \mathrm{m}$ on the pumped area to take snapshots of electron DPs at different time delays set by a linear translation stage. The mean free path of $88 \mathrm{keV}$ electrons is around $40 \mathrm{~nm}$ and limits the electron probe depth, i.e. the probed sample thickness, to about $100 \mathrm{~nm}$. Thus, for a $\sim 110 \mathrm{~nm}$ optical skin depth of $800 \mathrm{~nm}$ laser pulses on $\mathrm{La}_{0.84} \mathrm{Sr}_{0.16} \mathrm{MnO}_{3}$, comparable to the aforementioned probed sample thickness, the electron probed area can be assumed to be homogenously pumped, despite the sample's slightly wedged shape. In addition, we pre-set the sample base temperature well below the structure phase transition temperature by the heating-cooling stage and use a lower repetition rate of $100 \mathrm{~Hz}$, instead of regular $1 \mathrm{kHz}$, to minimize the increase of sample base temperature due to residual 
heating from the pump laser, ensuring that the sample is in the orthorhombic phase before time zero.

Fig. 1 (b) depicts a typical DP taken by FED with pump beam blocked. It is featured with a series of half-indexed diffraction spots between primary Bragg diffraction spots, similar to the reported static electron diffraction measurement at $85 \mathrm{~K},{ }^{13}$ indicating an orthorhombic crystal structure. Fig. 1 (c) and (d) display a magnified view of DPs of LSMO taken before and 1 ns after time zero, under a pump fluence of $1.5 \mathrm{~mJ} / \mathrm{cm}^{2}$ and at a pre-set sample base temperature of $273 \mathrm{~K}$. The DP before time zero is the same as that recorded without pump and as well as that in the reported static measurement at $85 \mathrm{~K}$, confirming that $\mathrm{La}_{0.84} \mathrm{Sr}_{0.16} \mathrm{MnO}_{3}$ is at the low temperature phase of orthorhombic structure. However, all the superlattice spots, marked by the arrows in the figure, disappear in the DP taken at 1 ns after time zero, consistent with the static electron DP at high temperature phase of rhombohedral structure. ${ }^{13}$ The disappearance of superlattice diffraction spots clearly indicated the destruction of orthorhombic structure after laser excitation, although the new structure could be rhombohedral or some other long-lived quasi-equilibrium phase with a pseudo cubic perovskite structure without the superlattice structure.

To understand the nature of the induced phase transition, we traced the temporal evolution of intensity in the primary Bragg spots as well as the superlattice spots, which is used as an ordering parameter of orthorhombic phase. At a pump fluence of $1.5 \mathrm{~mJ} / \mathrm{cm}^{2}$, a two-step intensity reduction as a function of time delay, an initial fast decay of $67 \%$ spot intensity in about 4 ps and a subsequent slower decrease of the remaining intensity in about $90 \mathrm{ps}$, can be seen clearly in the open circle curve in the Fig. 2 (a) and (b). We used a double exponential decay function to fit the intensity change and obtained the two corresponding decay time constants as $\tau_{1}=1.56 \pm 0.17 \mathrm{ps}$ and $\tau_{2}=30.2 \pm 5.8 \mathrm{ps}$, respectively. The intensity evolution of primary Bragg diffraction spots were analyzed, and the averaged intensity change of $(010)$ and $(0 \overline{10})$ spots as a function of time delay is shown in the inset of figure 2(a). Although constrained by a relatively high noise level, the intensity change of (010) spot displays a general trend of a fast drop of $7 \%$ in about 5 ps followed by a slow recovery with a final 
intensity overshoot about $5 \%$. It is worth pointing out that the amplitude change in the diffraction intensity of the primary Bragg diffraction spot is significantly smaller than that of half-indexed spots, indicating two distinct mechanisms for the two different structural dynamics. We attribute the primary Bragg intensity drop to the lattice disorder related to the lattice heating and photoinduced lattice disorder during the orthorhombic-to-rhombohedral phase transition.

We also studied the phase transition by varying the pump fluence while keeping the pre-set sample base temperature fixed at $273 \mathrm{~K}$, and the results are shown in Fig. 2 (a) and (b). In all these intensities curves, a similar bi-exponential feature with nearly the same time constant for the fast decay component $\left(\tau_{1}=1.56 \pm 0.17 \mathrm{ps}, 1.55 \pm 0.28 \mathrm{ps}\right.$, and $\left.1.48 \pm 0.19 \mathrm{ps}\right)$ and a fluence dependent slower component was observed. The time scale of the slow relaxation component increases with decreasing pump fluence, with time constants of $\tau_{2}=30 \pm 5.8 \mathrm{ps}, 102 \pm 32 \mathrm{ps}$, and $890 \pm 148 \mathrm{ps}$ for pump fluence of $1.50,1.13$ and $0.75 \mathrm{~mJ} / \mathrm{cm}^{2}$, respectively.

The amplitude of diffraction intensity change associated with the fast photoinduced process at different pump fluence is drawn in the inset of the Fig. 2 (b). It shows that suppression of superlattice structure is proportional to the pump fluence used in the current experiment: $67.2 \%$ for $1.5 \mathrm{~mJ} / \mathrm{cm}^{2}$, $51.9 \%$ for $1.12 \mathrm{~mJ} / \mathrm{cm}^{2}$ and $31.9 \%$ for $0.75 \mathrm{~mJ} / \mathrm{cm}^{2}$. The intercept point of fitting curve with fluence axis is very close to 0 , indicating the phase transition threshold is very low $\left(<0.2 \mathrm{~mJ} / \mathrm{cm}^{2}\right)$. Given the total number of photons absorbed and the pumped sample volume, it was estimated that roughly every $38 \mathrm{MnO}_{6}$ octahedrons absorb a single photon at a pump fluence of $1.5 \mathrm{~mJ} / \mathrm{cm}^{2}$, matching a cube with a side length of 3.4 octahedrons.

We carefully examined the effect of thermal heating on the sample and conclude that the thermal heating alone would be impossible to induce the observed structural phase transition in the LSMO sample. The heating of the LSMO sample after turning on the pump laser comes from two contributions: the residual heating $\left(\Delta T_{\text {res }}\right)$ and the transient pump heating $\left(\Delta T_{\text {pump }}\right)$. The residual heating $\left(\Delta T_{\text {res }}\right)$, which is the sample base temperature increase due to turning on the pump laser, was first measured by gradually changing the sample base temperature while keeping the pump fluence 
fixed at $1.5 \mathrm{~mJ} / \mathrm{cm}^{2}$. At the base temperature of $303 \mathrm{~K}$, the superlattice diffraction spots start to become weaker before the time zero, which implies that the residual heating $\Delta T_{\text {res }}$ is around $22 \mathrm{~K}$ at the $100 \mathrm{~Hz}$ repetition rate under the pump fluence of $1.5 \mathrm{~mJ} / \mathrm{cm}^{2}$. This is consistent with our estimation below. With the specific heat of $140 \mathrm{~J} / \mathrm{mol} \cdot \mathrm{K}$ for $\mathrm{LSMO}^{22}$ and optical the absorption coefficient of $\sim 9 \times 10^{4} \mathrm{~cm}^{-1}$ at $800 \mathrm{~nm},{ }^{23,24}$ an estimated temperature difference between the illuminated thin area and the surrounding unpumped thicker area (residue heating) is less than $20 \mathrm{~K}$. This is obtained by assuming that the absorbed energy of a pump pulse (50 fs, $100 \mathrm{~Hz})$ is evenly spread within the 10-ms-period (time interval from pulse to pulse), i.e., laser energy is absorbed by thin area and transported into thicker sample area at a constant rate. On the other hand, the temperature jump of the pumped area from a single pulse absorption $\left(\Delta T_{\text {pump }}\right)$ after time zero is about $33 \mathrm{~K}$, estimated by assuming all the absorbed energy had been converted into heat in the pumped region. The $\Delta T_{\text {res }}$ together with $\Delta T_{\text {pump }}$ raise the sample temperature from the pre-set $273 \mathrm{~K}$ to a maximum of $328 \mathrm{~K}$, which is just barely higher than the phase transition temperature of $325 \mathrm{~K}$. This temperature jump won't justify the observed 95\% intensity suppression of superlattice peaks, considering that latent heat must be supplied for the first order structural phase transition. We repeated the experiment at a lower pump fluence $0.75 \mathrm{~mJ} / \mathrm{cm}^{2}$ to quench the possible effect of thermal lattice heating. In this case, the expected sample maximum temperature would be $301 \mathrm{~K}$, well below the phase transition temperature, and the presented thermal heating would not be sufficient to trigger the transition. However, a $65 \%$ suppression of superlattice spot intensity was observed in that case. We also replicated the experiment at a lower pre-set sample temperature of $213 \mathrm{~K}$ while keeping pump fluence at $1.5 \mathrm{~mJ} / \mathrm{cm}^{2}$, once again a significant intensity drop of superlattice spot after time zero was observed, which could not be accounted by the lattice heating effect such as Debye-Waller effect. All these observations confirm that the observed phase transition is primarily induced by the photoexcitation, instead of subsequent thermal heating of lattice.

The bi-exponential relaxation reveals that the photoinduced melting of orthorhombic lattice ordering involves two distinct mechanisms with different time scales. The initial fast decay can be attributed to the lattice transformation triggered by $\mathrm{d}-\mathrm{d}$ charger transfer transition after absorption 
$1.55-\mathrm{eV}$ photons. In simple words, the photo-carriers break the orthorhombic symmetry, and drive the lattice to re-arrange towards to a higher symmetry in order to gain more kinetic energy. In the $\mathrm{AMO}_{3}$ perovskite structure, the mismatch of the equilibrium $\mathrm{A}-\mathrm{O}$ and $\mathrm{M}-\mathrm{O}$ bond lengths (measured by the deviation form unity of the tolerance factor) could cause cooperative rotations of $\mathrm{MnO}_{6}$ octahedrons, rotations about a cubic [111] axis give rhombohedral $(R \overline{3} c)$ symmetry and rotations about the [110] axis give orthorhombic (Pbnm) symmetry. ${ }^{25,26}$ Previous studies suggest that $\mathrm{MnO}_{6}$ octahedrons in orthorhombic LSMO also suffer incoherent or coherent Jahn-Teller distortion (JTD) due to the degeneracy of $e_{g}$ levels at $\mathrm{Mn}^{3+}$ sites. ${ }^{27,28}$ By means of JTD, Mn-O bonds extend in one direction and shrink in another direction. The ordering of JTD superimposes and strengthens the orthorhombic structure induced by bond length mismatch, and this orthorhombic structure is labeled as O'; the one associated with dynamic Jahn-Teller distortion is labelled as O*. ${ }^{25,29}$ Optical excitations at $800 \mathrm{~nm}$ $(1.55 \mathrm{eV})$ are resonant with the transition energy from the lower $e_{g}$ level $\left(3 \mathrm{~d}_{3 \mathrm{z}^{\wedge}-\mathrm{r}^{\wedge} 2}\right)$ to the higher $e_{g}$ level $\left(3 \mathrm{~d}_{\mathrm{x}^{\wedge} 2-\mathrm{y}^{\wedge} 2}\right)$ (Transition I) and/or a neighboring non-split $e_{g}$ level (Transition II), ${ }^{30,31}$ as shown schematically in Fig. 2 (c). As a result, the initial conditions for the existence of dynamic JTD are destroyed after absorbing 800-nm photons. The suppression of dynamic JTD straightens the Mn-O$\mathrm{Mn}$ bonds and equalizes the $\mathrm{Mn}-\mathrm{O}$ bond length along three directions. ${ }^{25}$ This will melt the $\mathrm{O} *$ phase imposed and strengthened by JTD, as the tolerance factor in photoexcited $\mathrm{O}^{*}$ phase has been very close to the critical value of orthorhombic-to-rhombohedral. ${ }^{27}$

The second slower decay of superlattice reflection can be associated with the subsequent further depopulation of the orthorhombic phase to fuel the growth and expansion of the newly formed domains of rhombohedral phase via phase front propagation. ${ }^{32}$ Such process could be collectively driven by several mechanisms, including, for example, lattice thermal excitation energized by the scattering of hot electrons with lattice, as well as the release of the excessive elastic energy from the newly formed domains to the remaining orthorhombic sites. Higher pump fluence will yield a higher lattice temperature and elastic energy density, consequently resulting in a faster progression of transformation, as shown in the pump-intensity dependent time constants observed in this study.

It is important to emphasize that two types of JTDs (the distortion of $\mathrm{MnO}_{6}$ from perfect 
octahedron), static and dynamic, present in the CMR materials. The former is long range and static, directly revealed by conventional static (time averaged) structural measurements, it is stable and robust. In contrast, dynamic JTD, which exist in the $\mathrm{La}_{0.84} \mathrm{Sr}_{0.16} \mathrm{MnO}_{3}$ sample used in this study, is local and dynamic, ${ }^{19}$ fluctuating in space or time or both, hard to be detected by time averaged structure measurements. As a result of these unique characteristics, dynamic JTD can be much easier to quench, consistent with our observation of very small threshold pump fluence. This low threshold is due to a very short coherent length of dynamic JTD, producing a low energy barrier for the O-R phase transition in $\mathrm{La}_{0.84} \mathrm{Sr}_{0.16} \mathrm{MnO}_{3}$, so that a single electron excitation could induce a local lattice transformation within the nearby octahedrons surrounding the excitation center. Decreasing the pumping flunence or photon number will not alter this process but only increasing the distance between these excitation centers. Therefore, the time-constant of the fast process remains unchanged, while the super-lattice intensity change will decrease linearly the pumping flunence.

To further attest our proposed correlation between orthorhombic-to-rhombohedral phase transition and the suppression dynamical JTD, we repeat the FED measurements on a non-doped $\mathrm{LaMnO}_{3}$, where a long-range static JTD and $\mathrm{O}^{\prime}$ structure exists at room temperature. Its structure phase transition from $\mathrm{O}^{\prime}$ to $\mathrm{R}$ happens at $1010 \mathrm{~K} \cdot{ }^{33}$ Under a similar excitation density and sample base temperature setting, within our detection limit, no change in the intensity of superlattice spots were observed in the entire time duration of $1.5 \mathrm{~ns}$ after time zero under the a pump fluence of $3.0 \mathrm{~mJ} / \mathrm{cm}^{2}$, which will result in same photon excitation density as $1.5 \mathrm{~mJ} / \mathrm{cm}^{2}$ on $\mathrm{La}_{0.84} \mathrm{Sr}_{0.16} \mathrm{MnO}_{3}$ by considering its absorption coefficient at $1.55 \mathrm{eV}$ is about half. ${ }^{34}$ This confirms that $\mathrm{O}$ ' structure with static JTD is much more robust to the $1.55 \mathrm{eV}$ photoexcitation than $\mathrm{O}^{*}$ phase with dynamical JTD ${ }^{33}$ Future study could further differentiate if this is due to the static JTD in $\mathrm{LaMnO}_{3}$ is too strong to be broken or the tolerance factor is far from the critical value of orthorhombic-to-rhombohedral transition and could not be tuned much under the current pump fluence.

In summary, we induced a structure phase transition in $\mathrm{La}_{0.84} \mathrm{Sr}_{0.16} \mathrm{MnO}_{3}$ with $1.55 \mathrm{eV}$ photons and resolved the two-step feature of phase transition. This study provided insight into structure evolution of CMR materials upon photon excitation and potential manipulation of their properties in the 
application of information storage. The direct and real time probe of photoinduced structure transition in LSMO also paves the way to investigate the dynamics of photo-induced phase transitions in many other correlated systems, such as charge transfer crystals, ${ }^{35-37}$ transition metal-oxides ${ }^{38,39}$ and spin crossover complexes, ${ }^{40,41}$ and ultimately unravel the mechanisms governing these transition dynamics.

We would like to acknowledge Steve McGill and Pedro U. Schlottmann for helpful discussions.This work is supported by National Science Foundation Grant No. 1207252. 
Figures and captions

\section{Figure 1}

(a)

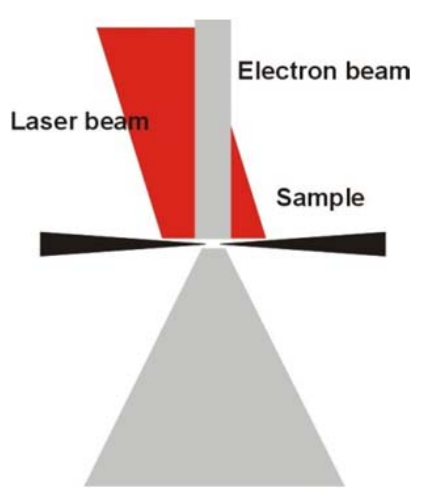

(c)

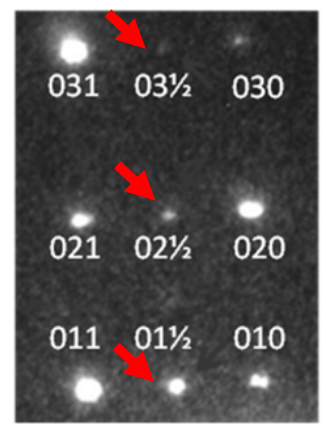

(b)

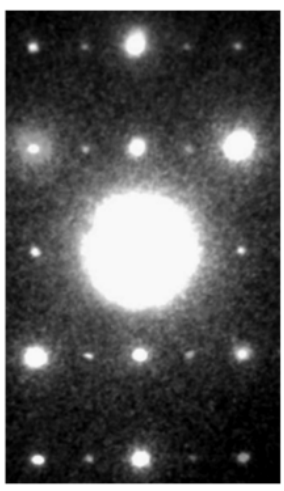

(d)

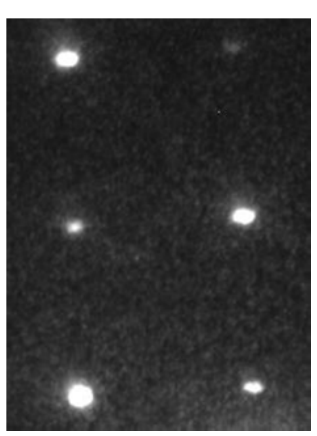

Figure 1 (a) Geometrical arrangement of the pump-probe pulses and the LSMO sample. Snapshots of diffraction pattern of $\mathrm{La}_{0.84} \mathrm{Sr}_{0.16} \mathrm{MnO}_{3}$ taken with pump laser blocked (b), with pump laser before (c) and at $1 \mathrm{~ns}$ after the time zero (d). The pump fluence is set at $1.5 \mathrm{~mJ} / \mathrm{cm}^{2}$ and the sample base temperature is $273 \mathrm{~K}$. 
Figure 2

(a)

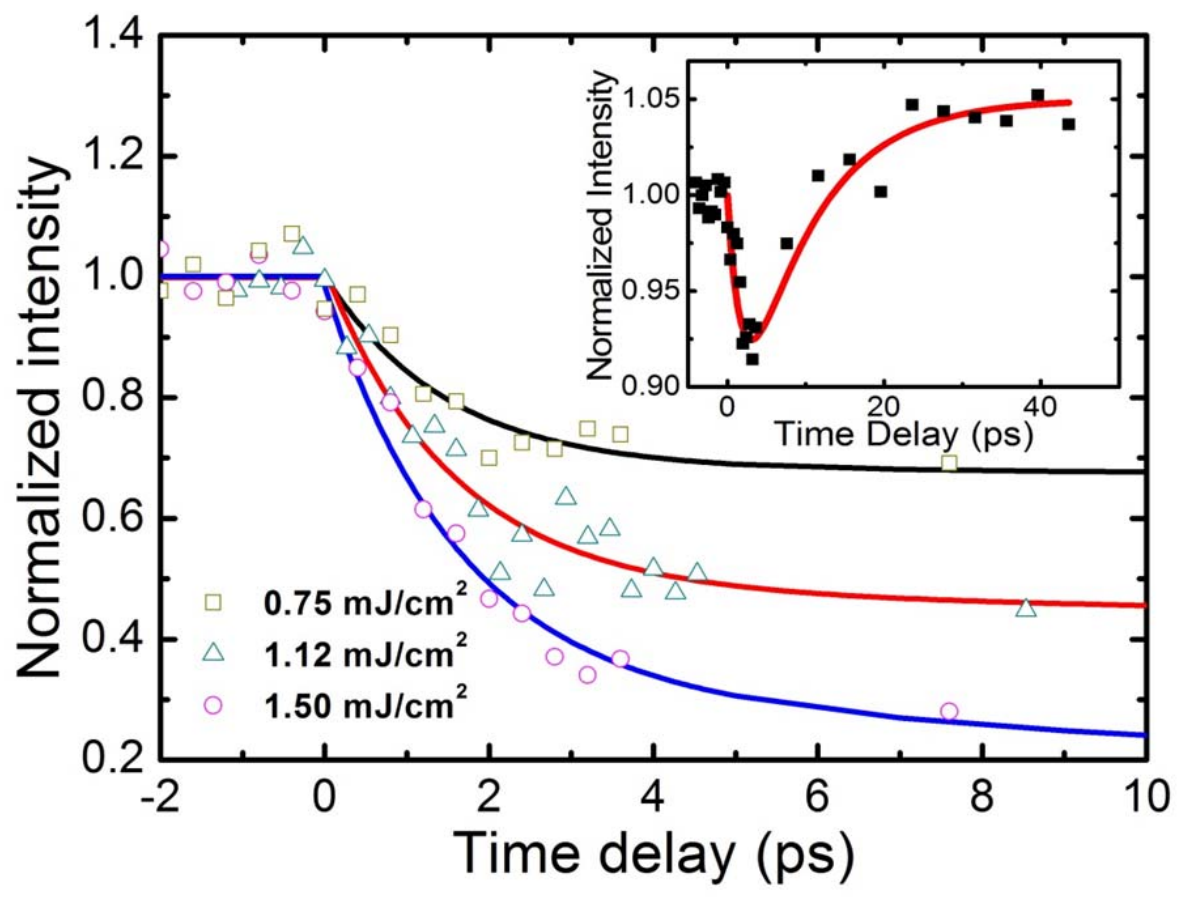

(b)

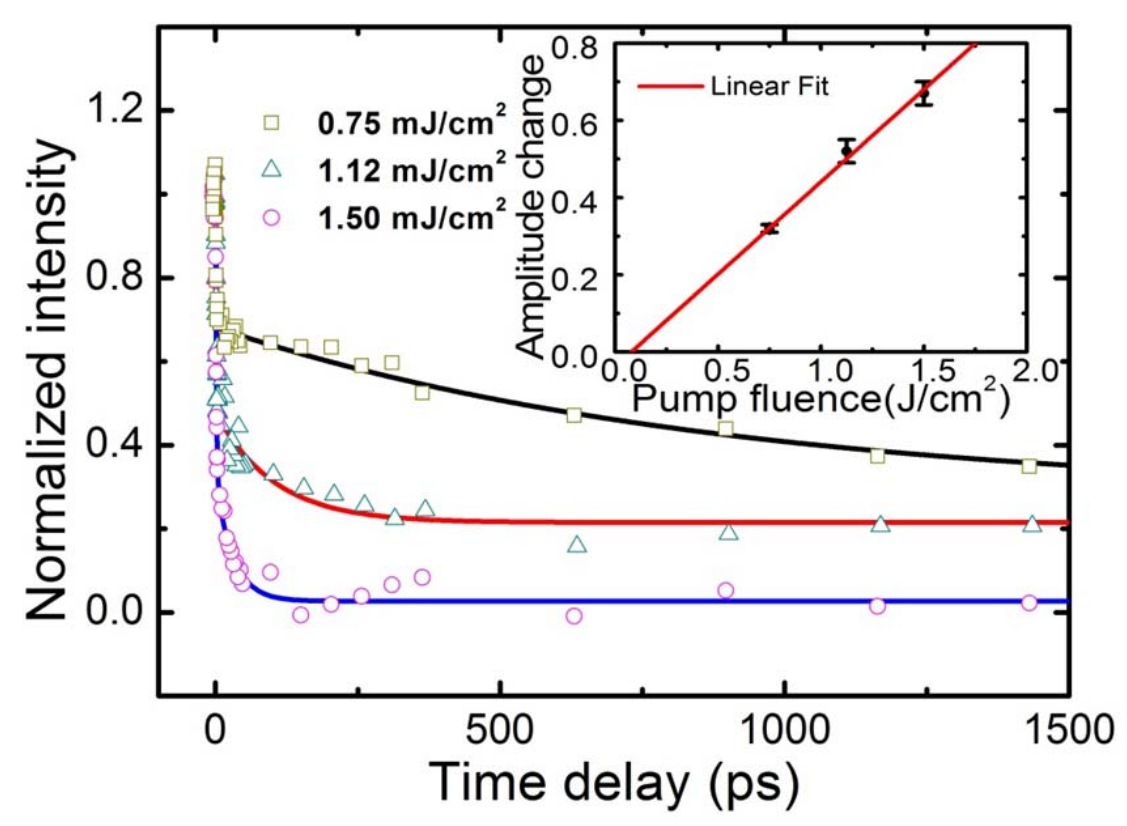


(c)

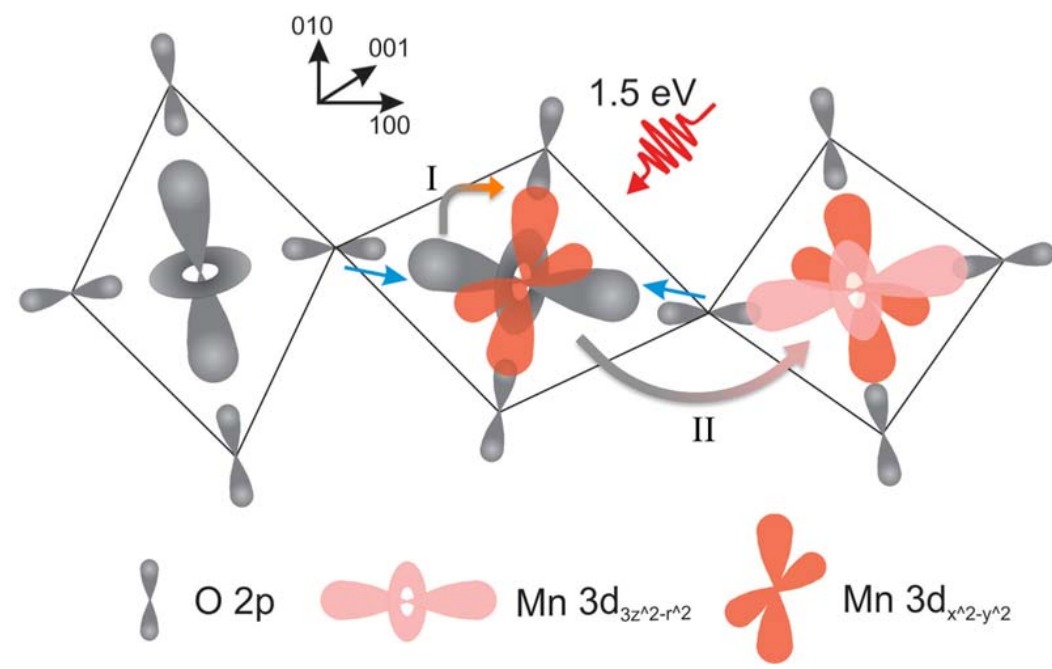

Figure 2 Temporal evolution of the normalized diffraction intensity of superlattice spots during (a) the first $10 \mathrm{ps}$ and (b) $1500 \mathrm{ps}$ after time zero at different pump fluence. The solid curves are bi-exponential fitting results. (c) Diagram of electronic orbitals involved in the photo-excitation. The grey orbitals are initially occupied, the pink orbital is occupied at the $\mathrm{Mn}^{3+}$ sites but unoccupied at $\mathrm{Mn}^{4+}$ sites, and the red orbitals are the possible final states during the transitions. Inset: (a) Intensity change of primary Bragg peak (b) amplitude of intensity change as a function of pump fluence for the fast relaxation process. 


\section{Figure 3}

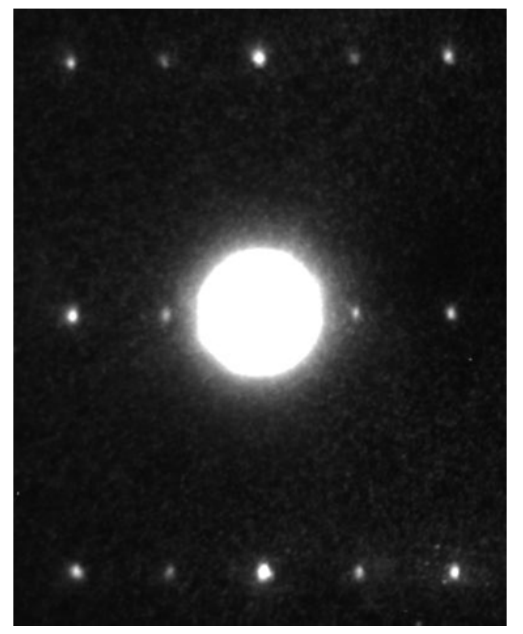

(a)

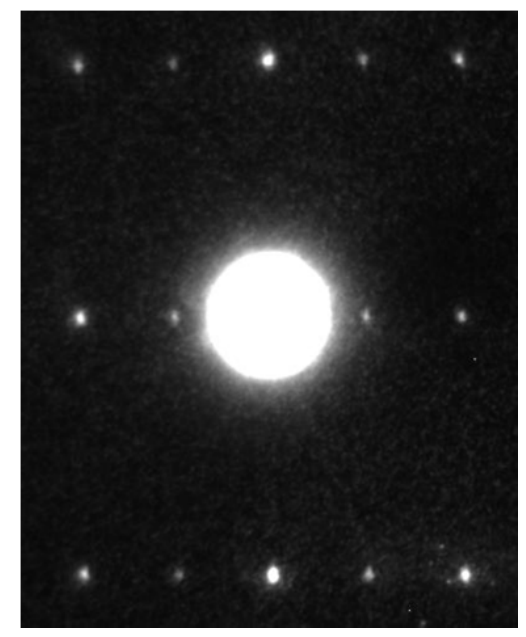

(b)

Fig. 3. Snapshots of diffraction pattern of $\mathrm{LaMnO}_{3}$ taken (a) before and (b) at $40 \mathrm{ps}$ after the time zero, at $3.0 \mathrm{~mJ} / \mathrm{cm}^{2}$ pump fluence and at $273 \mathrm{~K}$ base temperature. 


\section{References}

${ }^{1}$ G.H. Jonker and J.H. Van Santen, Physica 16, 337 (1950).

${ }^{2}$ R.M. Kusters, J. Singleton, D.A. Keen, R. McGreevy, and W. Hayes, Physica B 155, 362 (1989).

${ }^{3}$ A. Asamitsu, Y. Moritomo, Y. Tomioka, T. Arima, and Y. Tokura, Nature 373, 407 (1995).

${ }^{4}$ A. Millis, B. Shraiman, and R. Mueller, Phys. Rev. Lett. 77, 175 (1996).

5 P.G. Radaelli, D.E. Cox, M. Marezio, S.W. Cheong, P.E. Schiffer, and A.P. Ramirez, Phys. Rev. Lett. 75, 4488 (1995).

${ }^{6}$ S.J.L. Billinge, R.G. DiFrancesco, G.H. Kwei, J.J. Neumeier, and J.D. Thompson, Phys. Rev. Lett. 77, 715 (1996).

7 C. Zener, Phys. Rev. 81, 440 (1951).

8 M. Fiebig, K. Miyano, Y. Tomioka, and Y. Tokura, Journal of Luminescence 89, 87 (2000).

${ }^{9}$ R. Averitt, A. Lobad, C. Kwon, S. Trugman, V. Thorsmølle, and a. Taylor, Phys. Rev. Lett. 87, 017401 (2001).

${ }^{10}$ A. Cavalleri, T. Dekorsy, H.H.W. Chong, J.C. Kieffer, and R.W. Schoenlein, Phys. Rev. B 70, 1 (2004).

11 M. Rini, R. Tobey, N. Dean, J. Itatani, Y. Tomioka, Y. Tokura, R.W. Schoenlein, and A. Cavalleri, Nature 449, 72 (2007).

12 A. Urushibara, T. Arima, A. Asamitsu, G. Kido, and Y. Tokura, Phys. Rev. B 51, 14103 (1995).

13 M. Arao, Y. Koyama, Y. Inoue, and Y. Moritomo, Phys. Rev. B 62, 5399 (2000).

${ }^{14}$ P. Radaelli, M. Marezio, H. Hwang, S.-W. Cheong, and B. Batlogg, Phys. Rev. B 54, 8992 (1996).

15 A. Glazer, Acta Crystallographica Section B-Structural Science 28, 3384 (1972).

${ }^{16}$ H. Park, X. Wang, S. Nie, R. Clinite, and J. Cao, Solid State Commun. 136, 559 (2005).

17 N. Gedik, D.-S. Yang, G. Logvenov, I. Bozovic, and A.H. Zewail, Science 316, 425 (2007).

18 M. Eichberger, H. Schäfer, M. Krumova, M. Beyer, J. Demsar, H. Berger, G. Moriena, G. Sciaini, and R.J.D. Miller, Nature 468, 799 (2010).

19 J.-S. Zhou and J.B. Goodenough, Phys. Rev. B 64, 024421 (2001).

20 G.-L. Liu, J.-S. Zhou, and J.B. Goodenough, Phys. Rev. B 64, 144414 (2001).

21 S. Nie, X. Wang, J. Li, R. Clinite, and J. Cao, Microsc. Res. Tech. 72, 131 (2009).

22 D. Kim, B. Zink, F. Hellman, and J. Coey, Phys. Rev. B 65, 214424 (2002).

${ }^{23}$ A.I. Lobad, R.D. Averitt, C. Kwon, and A.J. Taylor, Appl. Phys. Lett. 77, 4025 (2000).

${ }^{24}$ N.N. Loshkareva, Y.P. Sukhorukov, E. A. Neifel'd, V.E. Arkhipov, A. V. Korolev, V.S. Gaviko, E. V. Panfilova, V.P. Dyakina, Y.M. Mukovskii, and D. a. Shulyatev, J. Exp. Theor. Phys. 90, 389 (2000).

25 J.B. Goodenough, in Localized to Itiner. Electron. Transit. Perovskite Oxides, edited by J.B.

Goodenough (Springer Berlin Heidelberg, 2001), pp. 1-16

${ }^{26}$ H. Hwang, S.-W. Cheong, P. Radaelli, M. Marezio, and B. Batlogg, Phys. Rev. Lett. 75, 914

(1995).

27.H. Kawano, R. Kajimoto, M. Kubota, and H. Yoshizawa, Phys. Rev. B 53, R14709 (1996).

28 J.B. Goodenough, Reports Prog. Phys. 67, 1915 (2004).

29 I.B. Bersuker, The Jahn-Teller Effect (Cambridge University Press, 2006).

30 M. Quijada, J. Černe, J. Simpson, and H. Drew, Phys. Rev. B 58, 16093 (1998).

31 J. Jung, K. Kim, T. Noh, E. Choi, and J. Yu, Phys. Rev. B 57, R11043 (1998).

32 K. Nasu, Photoinduced Phase Transition (World Scientific Publishing, 2004).

33 J. Rodriguez-Carvajal and M. Hennion, Phys. Rev. B 57, 3189 (1998).

34 Y. Okimoto, T. Katsufuji, T. Ishikawa, T. Arima, and Y. Tokura, Phys. Rev. B 55, 4206 (1997). 
35 R.S. Potember, T.O. Poehler, and R.C. Benson, Appl. Phys. Lett. 41, 548 (1982).

36 S. Koshihara, Y. Tokura, T. Mitani, G. Saito, and T. Koda, Phys. Rev. B 42, 6853 (1990).

37 E. Collet, M.-H. Lemée-Cailleau, M. Buron-Le Cointe, H. Cailleau, M. Wulff, T. Luty, S.-Y. Koshihara, M. Meyer, L. Toupet, P. Rabiller, and S. Techert, Science 300, 612 (2003).

38 M. Fiebig, K. Miyano, Y. Tomioka, and Y. Tokura, Science 280, 1925 (1998).

39 A. Cavalleri, C. Tóth, C. Siders, J. Squier, F. Ráksi, P. Forget, and J. Kieffer, Phys. Rev. Lett. 87, 237401 (2001).

40 P. Gütlich, A. Hauser, and H. Spiering, Angew. Chemie Int. Ed. English 33, 2024 (1994).

41 Y. Ogawa, S. Koshihara, K. Koshino, T. Ogawa, C. Urano, and H. Takagi, Phys. Rev. Lett. 84, 3181 (2000). 


\section{Electron beam}

(b) $\begin{array}{llll}021 & 020 & 02-1\end{array}$

Sample

$011 \quad 010 \quad 01-1$

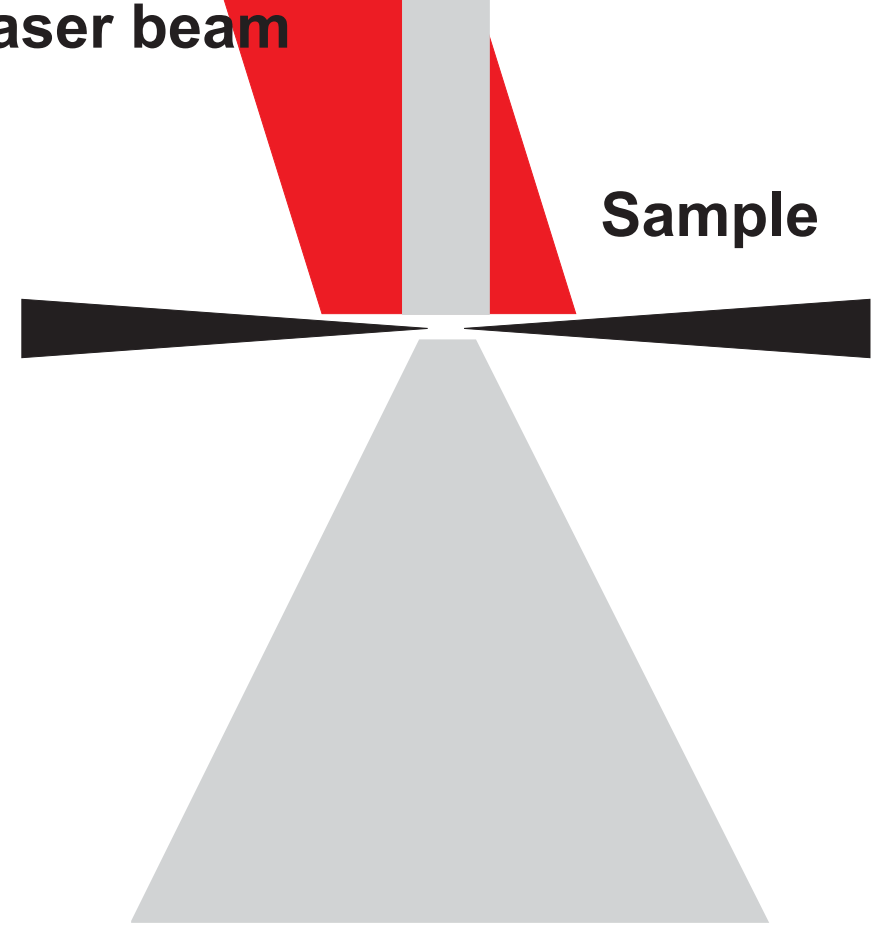

(c)

$031 \quad 03 \frac{1}{2} \quad 030$

(d)

$\begin{array}{lll}021 & 021 / 2 \quad 020\end{array}$

$011 \quad 011 / 2 \quad 010$ 


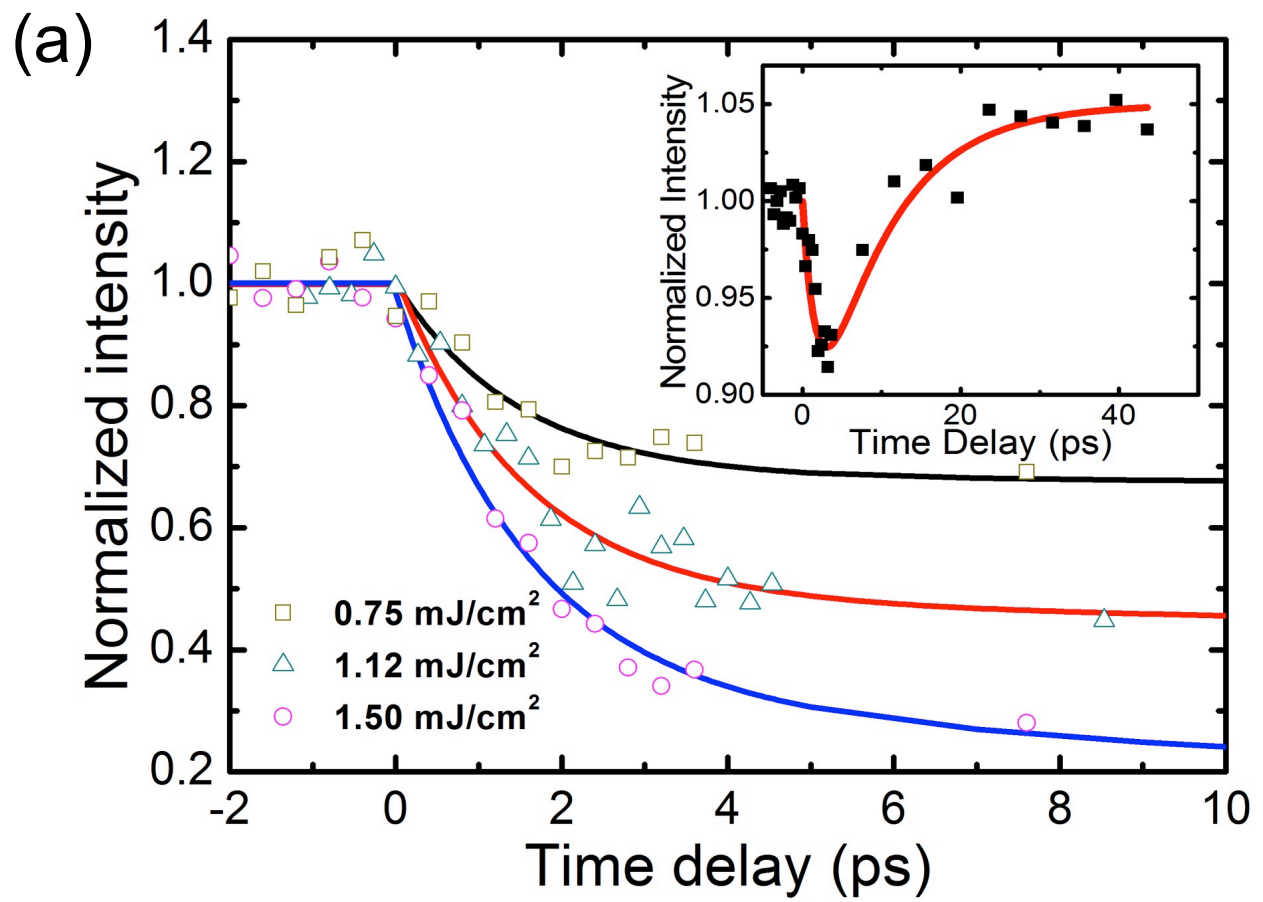

(b)

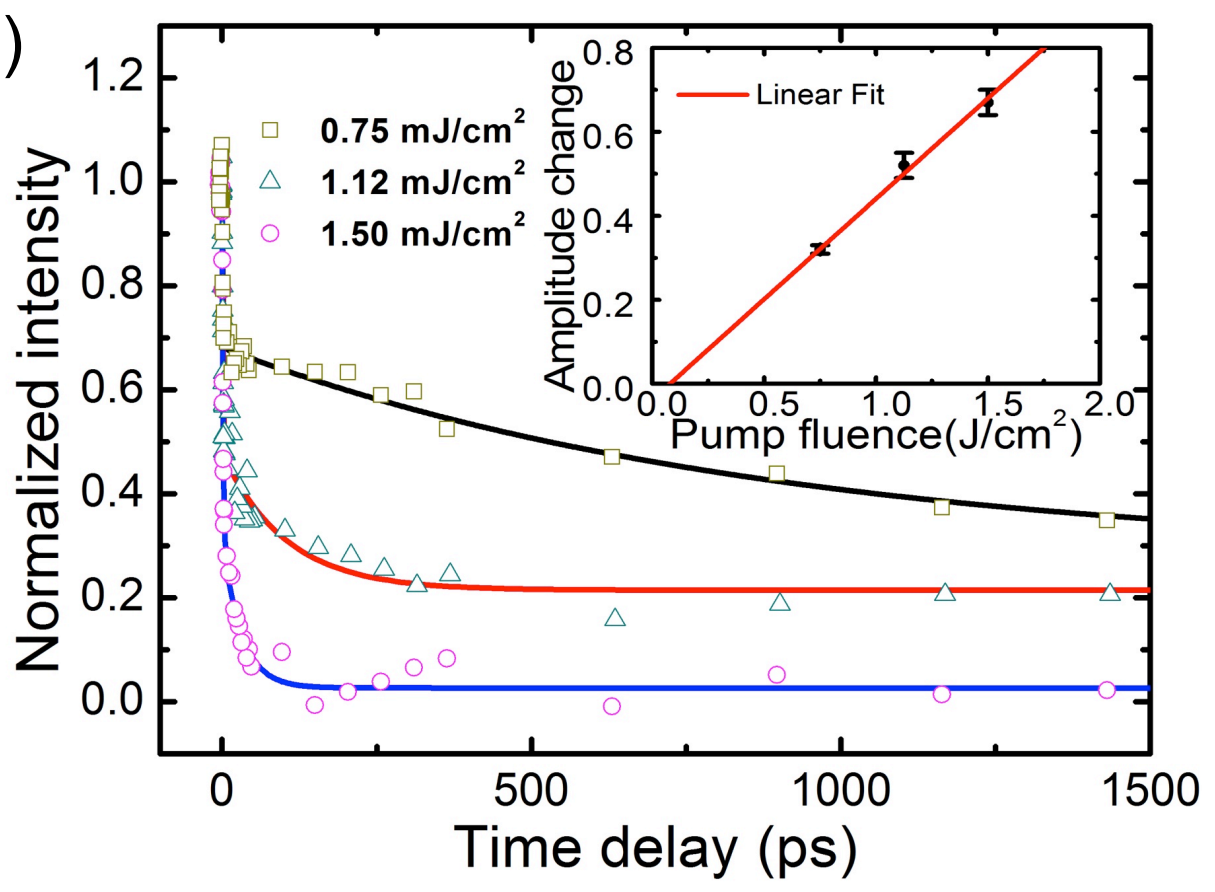

(c)

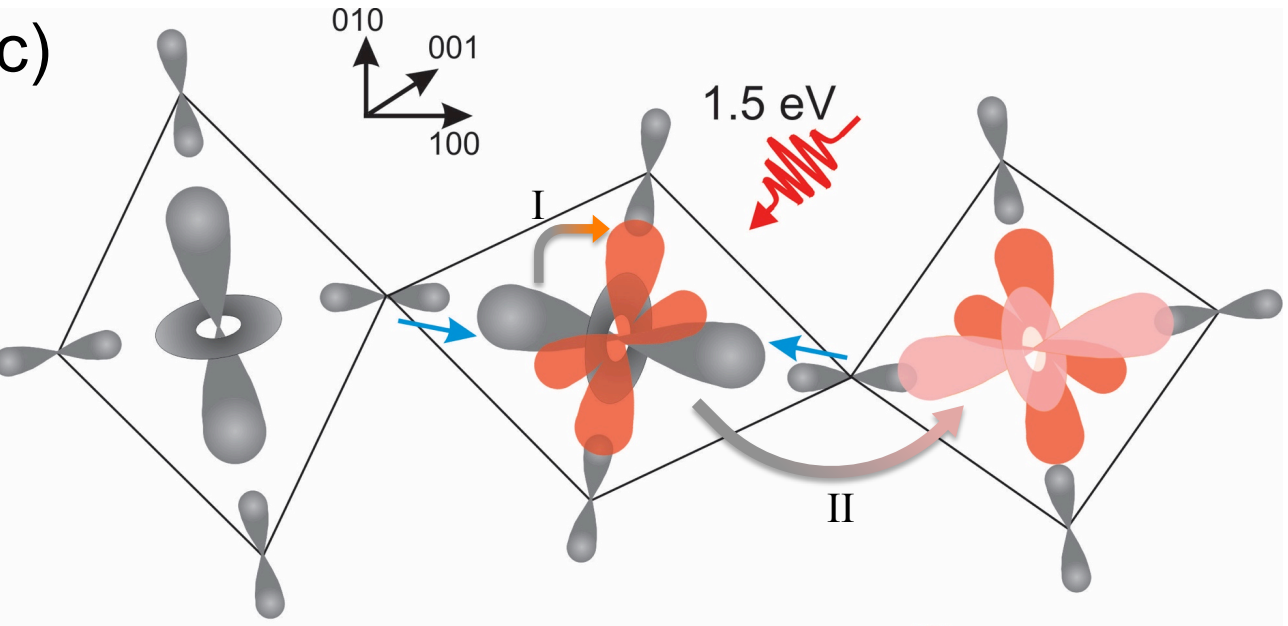



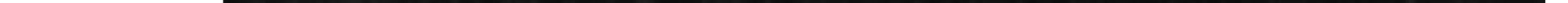